\title{
MULTIPLICATION OPERATORS ON WEIGHTED SPACES IN THE NON-LOCALLY CONVEX FRAMEWORK
}

\author{
L. A. KHAN \\ Department of Mathematics \\ Quaid-i-Azam University \\ Islamabad - 45320, PAKISTAN
}

A. B. THAHEEM

Department of Mathematical Sciences

King Fahd University of Petroleum and Minerals

Mail Box 469, Dhahran 31261, SAUDI ARABIA

(Received December 13, 1994 and in revised form June 21, 1995)

\begin{abstract}
Let $X$ be a completely regular Hausdorff space, $E$ a topological vector space, $V$ a Nachbin family of weights on $X$, and $C V_{0}(X, E)$ the weighted space of continuous $E$-valued functions on $X$. Let $\theta: X \rightarrow C$ be a mapping, $f \in C V_{0}(X, E)$ and define $M_{\theta}(f)=\theta f$ (pointwise). In case $E$ is a topological algebra, $\psi: X \rightarrow E$ is a mapping then define $M_{\psi}(f)=\psi f$ (pointwise). The main purpose of this paper is to give necessary and sufficient conditions for $M_{\theta}$ and $M_{\psi}$ to be the multiplication operators on $C V_{0}(X, E)$ where $E$ is a general topological space (or a suitable topological algebra) which is not necessarily locally convex. These results generalize recent work of Singh and Manhas based on the assumption that $E$ is locally convex.
\end{abstract}

KEY WORDS AND PHRASES: Nachbin family of weights, topological vector spaces, vector-valued continuous functions, weighted topology, multiplication operators, locally idempotent topological algebras.

1991 AMS SUBJECT CLASSIFICATION CODES: 47B38, 46E40, 46A16.

\section{INTRODUCTION}

The fundamental work on weighted spaces of continuous scalar-valued functions has been done mainly by Nachbin $[9,10]$ in the 1960 's. Since then it has been studied extensively for a variety of problems such as weighted approximation, characterization of the dual space, approximation property, description of inductive limit and of tensor-product, etc for both scalar- and vector-valued functions (for instance see [1-5,8-14]). Recently Singh and Summers [13] have studied the notion of composition operators on $C V_{0}(X, C)$. Later, Singh and Manhas [12] made an analogous study of multiplication operators on $C V_{0}(X, E)$, assuming $E$ to be a locally convex space or a locally $m$-convex algebra. The purpose of this paper is to generalize the results of Singh and Manhas [12] to the case when $E$ is a general topological vector space which is not necessarily locally convex. Section 3 contains our main results while section 2 is devoted to some technical preliminaries required for the development of our results

\section{PRELIMINARIES}

Throughout this paper we shall assume, unless stated otherwise, that $X$ is a completely regular Hausdorff space and $E$ is a non-trivial Hausdorff topological vector space Let $S^{+}(X)$ denote the set of 
all non-negative upper-semicontinuous functions on $X$, and let $S_{0}^{+}(X)$ (respectively $S_{c}^{+}(X)$ ), be the subset of $S^{+}(X)$ consisting of those functions vanishing at infinity (respectively having compact support) A Nachbin family on $X$ is a subset $V$ of $S^{+}(X)$ such that, given $u, v \in V$, there exist $w \in V$ and $t>0$ so that $u, v \leq t w$ (pointwise); the elements of $V$ are called weights. Let $C(X, E)\left(C_{b}(X, E)\right)$ be the vector space of all continuous (and bounded) $E$-valued functions on $X$, and let $C V_{b}(X, E)\left(C V_{0}(X, E)\right)$ denote the subspace of $C(X, E)$ consisting of those $f$ such that $v f$ is bounded (vanishes at infinity) for each $v \in V$ When $E=C$ (or $R$ ), these spaces are denoted by $C(X), C_{b}(X), C V_{b}(X)$, and $C V_{0}(X)$ If $\phi \in C(X)$ and $a \in E$, then $\phi \otimes a$ is a function in $C(X, E)$ defined by $(\phi \otimes a)(x)=\phi(x) a(x \in X)$ If $U$ and $V$ are two Nachbin families on $X$ and, for each $u \in U$, there is a $v \in V$ such that $u \leq v$, then we write $U \leq V$. If, for each $x \in X$, there is a $v \in V$ with $v(x) \neq 0$, we write $V>0$. For any function $\theta: X \rightarrow C$, we let $V|\theta|=\{v|\theta|: v \in V\}$.

Given any Nachbin family $V$ on $X$, the weighted topology $w_{v}$ on $C V_{b}(X, E)$ is defined as the linear topology which has a base of neighborhoods of 0 consisting of all sets of the form

$$
N(v, G)=\left\{f \in C V_{b}(X, E):(v f)(X) \subseteq G\right\},
$$

where $v \in V$ and $G$ is a neighborhood of 0 in $E ; C V_{b}(X, E)$ endowed with $w_{v}$ is called a weighted space We mention that if $V=S_{0}^{+}(X)$, then $C V_{b}(X, E)=C V_{0}(X, E)=C_{b}(X, E)$ and $w_{v}=\beta$, the strict topology and write as $\left(C_{b}(X, E), \beta\right)$; if $V=S_{c}^{+}(X)$, then $C V_{b}(X, E)=C V_{0}(X, E)=C(X, E)$ and $w_{v}=k$, the compact-open topology and we write as $(C(X, E), k)$. For more information on weighted spaces, we refer to [1-2,9-14] when $E$ is a scalar field or a locally convex space and to [1,3$5,8]$ in the general setting.

Let $\theta: X \rightarrow C$ and $\psi: X \rightarrow E$ be two mappings, and let $L(X, E)$ be the vector space of all functions from $X$ into $E$. The scalar multiplication on $E$ and, in case $E$ is an algebra, multiplication on $E$ give rise to two linear mappings $M_{\theta}$ and $M_{\psi}$ from $C V_{b}(E, X)$ into $L(X, E)$ defined by $M_{\theta}(f)=\theta f$ and $M_{\psi}(f)=\psi f$, where the product of functions is defined pointwise. If $M_{\theta}$ and $M_{\psi}$ map $C V_{b}(X, E)\left(C V_{0}(X, E)\right)$ into itself and are continuous, they are called multiplication operators on $C V_{b}(X, E)\left(C V_{0}(X, E)\right)$ induced by $\theta$ and $\psi$, respectively.

A neighborhood $G$ of 0 in $E$ is called shrinkable if $r \bar{G} \subseteq$ int $G$ for $0 \leq r<1$. By ([6], Theorems 4 and 5), every Hausdorff topological vector space has a base of shrinkable neighborhoods of 0 and also the Minkowski functional $\rho_{G}$ of any such neighborhood $G$ is continuous.

Now let $E$ be a topological algebra with jointly continuous multiplication and having $W$, a base of neighborhoods of 0 . Then, given any $G \in W$, there exists an $H \in W$ such that $H^{2} \subseteq G$. (Here $H^{2}=\{a b: a, b \in H\}$.) A subset $G \in W$ is called idempotent (or multiplicative) if $G^{2} \subseteq G$ Following Zelazko ([16], p. 31), $E$ is said to be a locally idempotent algebra if it has a base of neighborhoods of 0 consisting of idempotent sets. It is easily seen that if $G \in W$ is idempotent, then $\rho_{G}$ is submultiplicative $\rho_{G}(a b) \leq \rho_{G}(a) \rho_{G}(b)$ for all $a, b \in E$; further, if $E$ has an identity $e, \rho_{G}(e) \geq 1$. The notion of locally idempotent algebras is a strict generalization of the notion of locally $m$-convex algebras introduced by Michael [7] (see also [15, p. 348]).

\section{CHARACTERIZATION OF MULTIPLICATION OPERATORS}

In this section, we give necessary and sufficient conditions for $M_{\theta}$ and $M_{\psi}$ to be the multiplication operators on the weighted space $C V_{0}(X, E)$. (These results hold also for the space $C V_{b}(X, E)$ with slight modification in the proofs and are therefore omitted.) To avoid trivial cases we assume that the Nachbin family $V$ on $X$ satisfies the following conditions

$\left(^{*}\right) V>0$;

${ }^{* *}$ ) corresponding to each $x \in X$, there exists an $h_{x} \in C V_{0}(X)$ such that $h_{x}(x) \neq 0$ (This holds in particular, when each $v$ in $V$ vanishes at infinity or $X$ is locally compact.)

THEOREM 3.1. For a mapping $\theta: X \rightarrow C$, the following are equivalent: 
(a) $\theta$ is continuous and $V|\theta| \leq V$;

(b) $M_{\theta}$ is a multiplication operator on $C V_{0}(X, E)$.

PROOF. Let $W$ be a base of closed, balanced, and shrinkable neighborhoods of 0 in $E$.

(a) $\Rightarrow$ (b). We first show that $M_{\theta}$ maps $C V_{0}(X, E)$ into itself. Let $f \in C V_{0}(X, E)$, and let $v \in V$ and $G \in W$ Choose $u \in V$ such that $v|\theta| \leq u$ There exists a compact set $K \subseteq X$ such that $u(x) f(x) \in G$ for all $x \in X \backslash K$ Then, since $G$ is balanced,

$$
v(x) M_{\theta}(f)(x)=v(x) \theta(x) f(x) \in G
$$

for all $x \in X \backslash K \quad$ Hence $v M_{\theta}(f)$ vanishes at infinity; further, since $\theta$ is continuous, $M_{\theta}(f) \in C V_{0}(X, E)$. To prove the continuity of $M_{\theta}$, let $\left\{f_{\alpha}\right\}$ be a net in $C V_{0}(X, E)$ with $f_{\alpha} \rightarrow 0$ Let $v, G$ and $u$ be chosen as above. Choose an index $\alpha_{0}$ such that $f_{\alpha} \in N(u, G)$ for all $\alpha \geq \alpha_{0}$ Then it follows that $\theta f_{\alpha} \in N(v, G)$ for all $\alpha \geq \alpha_{0}$. Thus $M_{\theta}\left(f_{\alpha}\right) \rightarrow 0$. So $M_{\theta}$ is continuous at 0 and hence, by linearity, it is continuous on $C V_{0}(X, E)$.

(b) $\Rightarrow$ (a). We first show that $\theta$ is continuous. Let $\left\{x_{\alpha}\right\}$ be a net in $X$ with $x_{\alpha} \rightarrow x \in X$ By assumption ( $\left.{ }^{* *}\right)$, there exists an $h \in C V_{0}(X)$ such that $h(x) \neq 0$ Since $M_{\theta}$ is a self-map on $C V_{0}(X, E)$, it follows that the function $\theta h$ from $X$ into $C$ is continuous. Hence $\theta\left(x_{\alpha}\right) h\left(x_{\alpha}\right) \rightarrow \theta(x) h(x)$ and consequently $\theta\left(x_{\alpha}\right) \rightarrow \theta(x)$. We next show that $V|\theta| \leq V$. Let $v \in V$ By continuity of $M_{\theta}$, given $G \in W$, there exist $u \in V$ and $H \in W$ such that

$$
M_{\theta}(N(u, H)) \subseteq(v, G) .
$$

Without loss of generality we may assume that $G \cup H$ is a proper subset of $E$. Choose $a \in X \backslash(G \cup H)$, and put $t=\rho_{H}(a) / \rho_{G}(a)$. We claim that $v|\theta| \leq 2 t u$ Fix $x_{0} \in X$. We shall consider two cases $u\left(x_{0}\right) \neq 0$ and $u\left(x_{0}\right)=0$.

Suppose that $u\left(x_{0}\right) \neq 0$, and let $\epsilon=u\left(x_{0}\right)$. Then $D=\{x \in X: u(x)<2 \epsilon\}$ is an open neighborhood of $x_{0}$. Using the complete regularity of $X$ and the assumption ( $\left.{ }^{* *}\right)$, there is an $h \in C V_{0}(X, E)$ with $0 \leq h \leq 1, h\left(x_{0}\right)=1$, and $h(X \backslash D)=0$. Define $f=(h \otimes a) / 2 \epsilon \rho_{H}(a)$. Since $\rho_{H}$ is homogeneous, for any $x \in X$,

$$
\rho_{H}(u(x) f(x))=u(x) h(x) / 2 \epsilon<1,
$$

by considering the cases $x \in D$ and $x \in X \backslash D$. Since $H=\left\{b \in E: \rho_{H}(b) \leq 1\right\}$, we have $f \in N(u, H)$. Hence, by (1), $\theta f \in N(v, G)$. This implies that, for any $x \in X$,

$$
\rho_{G}\left(\theta(x) v(x) h(x) a / 2 \epsilon \rho_{H}(a)\right) \leq 1,
$$

or $v(x) h(x)|\theta(x)| \leq 2 t \epsilon$. In particular, $v\left(x_{0}\right)\left|\theta\left(x_{0}\right)\right| \leq 2 t u\left(x_{0}\right)$.

Now suppose that $u\left(x_{0}\right)=0$ but $v\left(x_{0}\right)|\theta(x)|>0$. Put $\epsilon=v\left(x_{0}\right)\left|\theta\left(x_{0}\right)\right| / 2 t \quad$ Let $D=\{x \in X: u(x)<\epsilon\}$, and choose an $h \in C V_{0}(X)$ as above. Define $g=(h \otimes a) / \epsilon \rho_{H}(a)$. We easily have $g \in N(u, H)$ and hence $\theta g \in N(v, G)$. From this we obtain

$$
v\left(x_{0}\right)\left|\theta\left(x_{0}\right)\right| \leq t \epsilon=v\left(x_{0}\right)\left|\theta\left(x_{0}\right)\right| / 2,
$$

which is impossible unless $v\left(x_{0}\right)\left|\theta\left(x_{0}\right)\right|=0$. This completes the proof.

We next consider the case of the operator $M_{\psi}$.

THEOREM 3.2. Let $E$ be a Hausdorff locally idempotent algebra with identity $e$ and $W$ a base of neighborhoods of 0 . Then, for a mapping $\psi: X \rightarrow E$, the following are equivalent:

(a) $\psi$ is continuous and $V \rho_{G} \circ \psi \leq V$ for every $G \in W$.

(b) $M_{\psi}$ is a multiplication operator on $C V_{0}(X, E)$.

PROOF. We may assume that $W$ consists of closed, balanced, shrinkable, and idempotent sets 
(a) $\Rightarrow$ (b) We first show that $M_{\psi}$ maps $C V_{0}(X, E)$ into itself Let $f \in C V_{0}(X, E)$, and let $v \in V$ and $G \in W$. Choose $u \in V$ such that $V \rho_{G} \circ \psi \leq u$ There exists a compact set $K \subseteq X$ such that $u(x) f(x) \in G$ for all $x \in X \backslash K$. Since $\rho_{G}$ is submultiplicative, for any $x \in X \backslash K$, we have

$$
\rho_{G}(v(x) \psi(x) f(x)) \leq v(x) \rho_{G}(\psi(x)) \rho_{G}(f(x)) \leq u(x) \rho_{G}(f(x)) \leq 1 ;
$$

hence $M_{\psi}(f) \in C V_{0}(X, E)$. Using again the submultiplicativity of $\rho_{G}$, the continuity of $M_{\psi}$ follows in the same way as in the proof of Theorem 1 .

(b) $\Rightarrow$ (a). Let $\left\{x_{\alpha}\right\}$ be a net in $X$ such that $x_{\alpha} \rightarrow x \in X$. Choose an $h \in C V_{0}(X)$ with $h(x) \neq 0$ Since $M_{\psi}$ is a self-map on $C V_{0}(X, E)$, it follows that the function $\psi(h \otimes a)$ from $X$ into $E$ is continuous. Hence $h\left(x_{\alpha}\right) \psi\left(x_{\alpha}\right) \rightarrow h(x) \psi(x)$ and consequently $\psi\left(x_{\alpha}\right) \rightarrow \psi(x)$ This proves the continuity of $\psi$. Next, let $v \in V$ and $G \in W$. There exist $u \in V$ and $H \in W$ such that

$$
M_{\psi}(N(u, H)) \subseteq N(v, G) .
$$

Without loss of generality, we may assume that $H$ is a proper subset of $E$. We claim that $v \rho_{G} \circ \psi \leq 2 \rho_{H}(e) u$.

Fix $x_{0} \in X$ First assume that $u\left(x_{0}\right) \neq 0$, and let $\epsilon=u\left(x_{0}\right)$. Then $D=\{x \in X: u(x)<2 \epsilon\}$ is an open neighborhood of $x_{0}$, so there exists an $h \in C V_{0}(X)$ such that $0 \leq h \leq 1, h\left(x_{0}\right)=1$, and $h(X \backslash D)=0$. Define $f=(h \otimes e) / 2 \epsilon \rho_{H}(e)$. Then, for any $x \in X$,

$$
\left.\rho_{H}(u(x) f(x))=\rho_{H}(u(x) h(x) e) / 2 \epsilon \rho_{H}(e)\right) \leq 1 ;
$$

that is, $f \in N(u, H)$. Hence, by (2), $\psi f \in N(v, G)$. This implies that, for any $x \in X$,

$$
v(x) h(x) \rho_{G}(\psi(x)) \leq 2 \epsilon \rho_{H}(e) .
$$

In particular, $v\left(x_{0}\right) \rho_{G}\left(\psi\left(x_{0}\right)\right) \leq 2 \rho_{H}(e) u\left(x_{0}\right)$. Next suppose that $u\left(x_{0}\right)=0$, but $v\left(x_{0}\right) \rho_{G}\left(\psi\left(x_{0}\right)\right)>0$ Put $\epsilon=v\left(x_{0}\right) \rho_{G}\left(\psi\left(x_{0}\right)\right) / 2 \rho_{H}(e)$. Let $D=\{x \in X: u(x)<\epsilon\}$, and choose an $h \in C V_{0}(X)$ as above. Define $g=(h \otimes e) / \epsilon \rho_{H}(e)$. Then $g \in N(u, H)$, so by $(2), \psi g \in N(v, g)$. From this we obtain

$$
v\left(x_{0}\right) \rho_{G}\left(\psi\left(x_{0}\right)\right) \leq \rho_{H}(e) \epsilon=v\left(x_{0}\right) \rho_{G}\left(\psi\left(x_{0}\right)\right) / 2,
$$

which is impossible unless $v\left(x_{0}\right) \rho_{G}\left(\psi\left(x_{0}\right)\right)=0$. This completes the proof.

Finally, we apply the above results to the cases: $V=S_{c}^{+}(X)$ and $V=S_{0}^{+}(X)$ and obtain the following.

THEOREM 3.3.

(i) If $\theta: X \rightarrow C$ is a continuous mapping, then $M_{\theta}$ is a multiplication operator on $(C(X, E), k)$.

(ii) If $E$ is a Hausdorff locally idempotent algebra with identity $e$ and $\psi: X \rightarrow E$ a continuous mapping, then $M_{\psi}$ is a multiplication operator on $(C(X, E), k)$.

PROOF. (i) In view of Theorem 1, we only need to verify that $V|\theta| \leq V$, where $V=S_{c}^{+}(X)$. Let $v \in V$. Choose a compact set $K \subseteq X$ with $v(x)=0$ for all $x \in X \backslash K$. Let $s=\sup \{|\theta(x)|: x \in K\}$ and $t=\sup \{v(x): x \in K\}$, and let $u=s t \chi_{K}$. Then $u \in V$ and clearly $v(x)|\theta(x)| \leq u(x)$ for all $x \in X$.

(ii) Let $W$ be a base of neighborhoods of 0 in $E$ consisting of closed, balanced, shrinkable, and idempotent sets. In view of Theorem 2, we only need to verify that $V \rho_{G} \circ \psi \leq V$ for every $G \in W$, where $V=S_{c}^{+}(X)$. Let $v \in V$ and $G \in W$. Choose a compact set $K \subseteq X$ with $v(x)=0$ for all $x \in X \backslash K$. Let $s=\sup \left\{\rho_{G}(\psi(x)): x \in K\right\}$ and $t=\sup \{v(x): x \in K\}$, and let $u=s t \chi_{K}$. Then $u \in V$ and clearly $v(x) \rho_{G}(\psi(x)) \leq u(x)$ for all $x \in X$. This completes the proof of the theorem

REMARK. The above result need not hold for the space $\left(C_{b}(X, E), \beta\right)$. To see this, consider $X=R^{+}, E=C$, and $V=S_{0}^{+}(X)$ Let $\theta=\psi: X \rightarrow C$ be a mapping given by $\theta(x)=x^{2}(x \in X)$, and let $v \in V$ be given by $v(x)=\frac{1}{x}(x \in X)$. Then $v(x)|\theta(x)|=x$ for all $x \in X$. Since each $u \in V$ is a bounded function, $v|\theta| \leq u$ for every $u \in V$. Hence $V|\theta| \leq V$ does not hold and so, by Theorem $1, M_{\theta}$ 
is not a multiplication operator on $\left(C_{b}(X), \beta\right)$. The same is also true for the space $\left(C_{b}(X), u\right)$, where $u$ is the uniform topology, since $\beta \leq u$. However, if $\theta$ and $\psi$ are bounded continuous functions, then it is easily seen that $M_{\theta}$ and $M_{\psi}$ are always multiplication operators on $C V_{0}(X, E)$ for any Nachbin family $V$

ACKNOWLEDGMENT. One of the authors (A. B. Thaheem) wishes to acknowledge the support provided by King Fahd University of Petroleum and Minerals during this research.

\section{REFERENCES}

[1] BIERSTEDT, K.D., Gewichtete Raume stetiger vektorwertiger funktionen und das injektive tensorprodukt, I, J. Reine Angew. Math. 259 (1973), 186-210, II, J. Reine Angew. Math., 260 (1973), 133-146.

[2] BIERSTEDT, K.D., MEISE, R. and SUMMERS, W.H., A projective description of weighted inductive limits, Trans. Amer. Math. Soc. 272 (1982), 107-160.

[3] KHAN, L.A., The strict topology on a space of vector-valued functions, Proc. Edinburgh Math. Soc. 22 (1979), 35-41

[4] KHAN, L.A., Weighted topology in the non-locally convex setting, Mat. Vesnik 37 (1985), 189195.

[5] KHAN, L.A., On approximation in weighted spaces of continuous vector-valued functions, Glasgow Math. J. 29 (1987), 65-68.

[6] KLEE, V., Shrinkable neighborhoods in Hausdorff linear spaces, Math. Ann. 141 (1960), 281-285

[7] MICHAEL, E.A., Locally multiplicatively convex topological algebras, Mem. Amer. Math. Soc. 11 (1952).

[8] NAWROCKI, M., On weak approximation and convexification in weighted spaces of vector-valued continuous functions, Glasgow Math. J. 31 (1989), 59-64.

[9] NACHBIN, L., Weighted approximation for algebras and modules of continuous functions: real and self-adjoint complex cases, Ann. of Math. 81 (1965), 289-302.

[10] NACHBIN, L., Elements of Approximation Theory, Math. Studies 14 (D. Van Nostrand, Princeton, 1967).

[11] PROLLA, J.B., Weighted spaces of vector-valued continuous functions, Ann. Mat. Pura. Appl. 89 (1971), 145-158.

[12] SINGH, R.K. and MANHAS, J.B., Multiplication operators on weighted spaces of vector-valued continuous functions, J. Austral. Math. Soc. (Series A) 50 (1991), 98-107.

[13] SINGH, R.K. and SUMMERS, W.H., Composition operators on weighted spaces of continuous functions, J. Austral. Math. Soc. (Series A) 45 (1988), 303-319.

[14] SUMMERS, W.H., A representation theorem for biequicontinuous completed tensor products of weighted spaces, Trans. Amer. Math. Soc. 146 (1969), 121-131.

[15] ZELAZKO, W., On the locally bounded and $m$-convex topological algebras, Studia Math. 19 (1960), 333-358.

[16] ZELAZKO, W., Metric generalizations of Banach algebras, Rozprawy Matematyezne 47 (1965), Warszawa. 


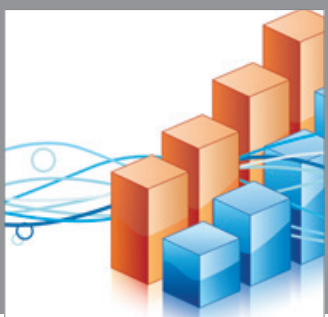

Advances in

Operations Research

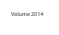

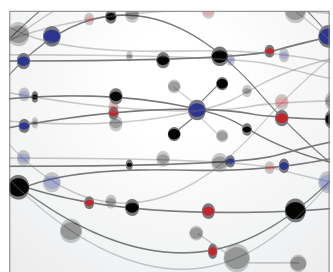

\section{The Scientific} World Journal
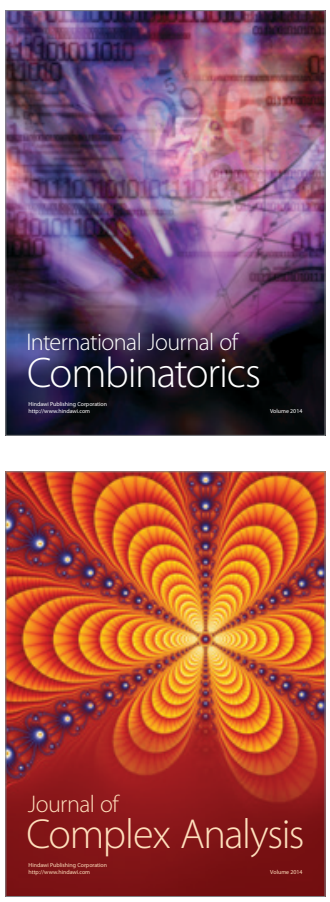

International Journal of

Mathematics and

Mathematical

Sciences
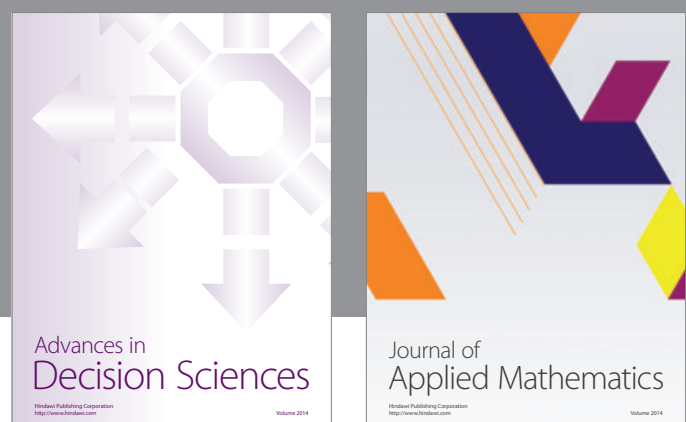

Journal of

Applied Mathematics
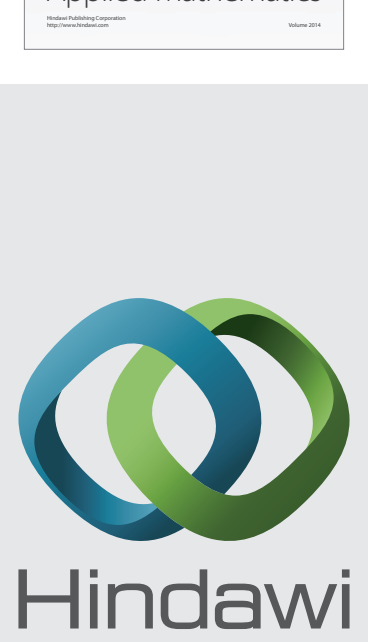

Submit your manuscripts at http://www.hindawi.com
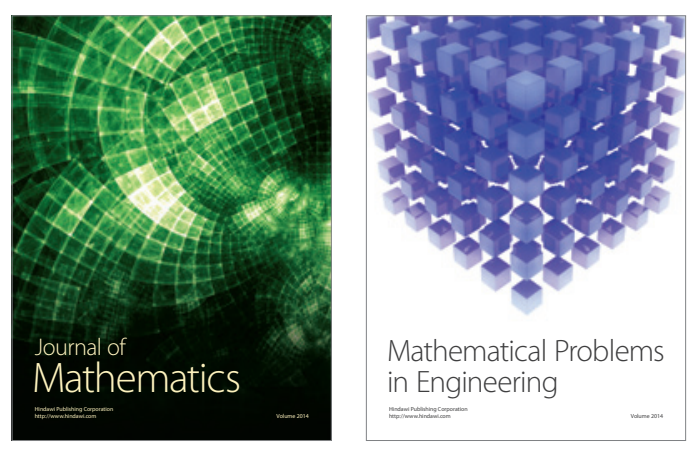

Mathematical Problems in Engineering
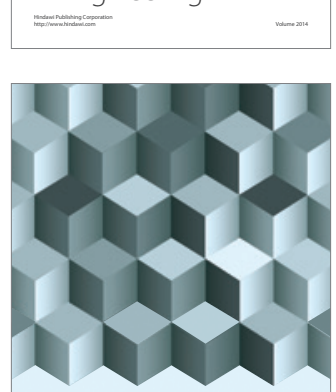

Journal of

Function Spaces
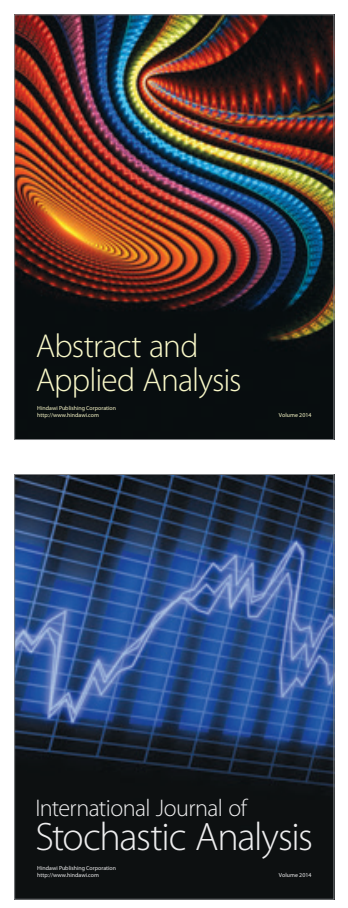

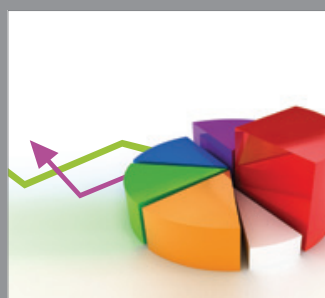

ournal of

Probability and Statistics

Promensencen
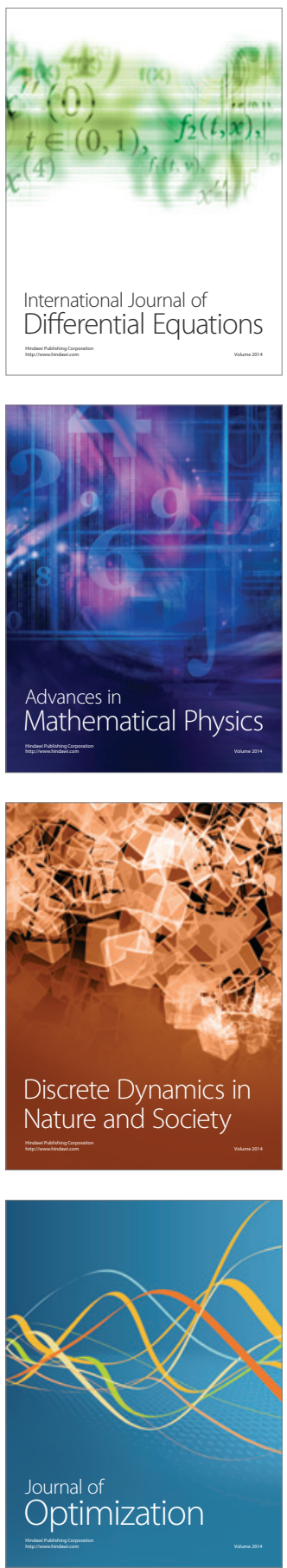